\title{
Ocorrência de corujas (Aves: Strigiformes) na RPPN Bugerkopf, Blumenau, Santa Catarina
}

\author{
Daniela Fink ${ }^{1 *}$ \\ Cláudia Sabrine Brandt ${ }^{2}$ \\ Adrian Eisen Rupp ${ }^{2}$ \\ Carlos Eduardo Zimmermann ${ }^{2,3}$ \\ ${ }^{1}$ PPG em Saúde e Meio Ambiente, Universidade da Região de Joinville - UNIVILLE \\ Laboratório de Ecosssistemas Costeiros, Unidade de São Francisco do Sul \\ Rodovia Duque de Caxias, s/n, Poste 128 - km 8, CEP 89240-000, São Francisco do Sul - SC, Brasil \\ ${ }^{2}$ Laboratório de Ecologia e Ornitologia, Instituto FURB de Pesquisas \\ Universidade Regional de Blumenau \\ Rua Antônio da Veiga, 140, CEP 89012-900, Blumenau - SC, Brasil \\ ${ }^{3}$ PPG em Ecologia e Conservação, Universidade Federal do Paraná, Curitiba - PR, Brasil \\ * Autor para correspondência \\ dani.fink@gmail.com
}

\section{Resumo}

A Ordem Strigiformes carece de estudos no país e especialmente no Estado de Santa Catarina. São raras as pesquisas que envolvem aspectos básicos como presença e ausência em áreas de Floresta Atlântica. $\mathrm{O}$ objetivo desta pesquisa foi inventariar a taxocenose de corujas (Aves: Strigiformes) na RPPN Bugerkopf, sul do município de Blumenau, Santa Catarina. O estudo foi realizado entre fevereiro de 2005 e maio de 2006, sendo os trabalhos de campo realizados em visitas mensais de duas a três noites, entre as 18:00 e 23:00h, somente com lua crescente ou cheia. Em um total de $69 \mathrm{~h}$ de estudo foram registradas as seguintes espécies: Tyto alba, Megascops choliba, Megascops sanctaecatarinae, Glaucidium minutissimum, Pulsatrix koeniswaldiana e Asio stygius. A espécie mais detectada foi G. minutissium, enquanto $M$. choliba e $P$. koeniswaldiana foram as espécies com menor registro neste estudo.

Palavras-chave: Brasil; Corujas; Mata Atlântica; Santa Catarina; Strigiformes

\section{Abstract}

Occurence of owls (Birds: Strigiformes) in the RPPN Bugerkopf reserve in Blumenau, Santa Catarina. The order Strigiformes is a poorly known group in Brazil, especially in Santa Catarina, and basic surveys of owls in the Atlantic Forests are scarce. The aim of this study was to inventory the owls of RPPN Bugerkopf, a private reserve in Blumenau, Santa Catarina. The study was conducted between February 2005 and May 2006. Fieldwork was carried out during monthly visits, for two to three nights when there was a crescent or full moon, between the hours of 18:00 and 23:00. In a total of 69 study hours, the following species were observed: Tyto alba, Megascops choliba, Megascops sanctaecatarinae, Glaucidium minutissimum, Pulsatrix koeniswaldiana and Asio stygius. The species most frequently detected was G. minutissimum, while M. choliba and $P$. koeniswaldiana were recorded the least.

Key words: Atlantic Forest; Brazil; Owls; Santa Catarina; Strigiformes 


\section{Introdução}

A ordem Strigiformes (corujas, mochos e caburés) apresenta aproximadamente 250 espécies distribuídas em todos os continentes, com exceção da Antártida (KÖNIG; WEICK, 2008). Na região Neotropical há 85 espécies registradas, das quais 23 ocorrem no Brasil (KÖNIG; WEICK, 2008; CBRO, 2011). Porém, apesar da importância ecológica destes rapinantes, poucos estudos foram desenvolvidos com Strigiformes no Brasil (SILVA et al., 2002).

Os estudos com aves noturnas como as corujas é desafiador, visto que as metodologias e informações ainda são muito escassas (BORGES et al., 2004). Como os procedimentos comumente utilizados em aves diurnas são ineficientes, o playback de vocalizações representa o método mais usado para estudar Strigiformes (BRAGA, 2006). Deste modo, os registros de corujas são normalmente realizados em levantamentos visuais/ auditivos de aves em geral, captura em redes de neblina, ou registros de indivíduos atropelados (BENCKE; BENCKE, 1999). Assim, poucos estudos tratam especificamente de aves noturnas, como as corujas (e.g. AZEVEDO et al., 2003; AMARAL, 2007).

Nos últimos anos a ornitologia catarinense teve um avanço considerável, e alguns registros importantes de aves noturnas foram publicados (AZEVEDO et al., 2003; PIACENTINI et al., 2006; RUPP et al., 2007; LEGAL et al., 2009). No entanto, as aves noturnas ainda carecem de informações básicas, como uso do habitat, abundância, dieta, comportamento reprodutivo, sendo continuadamente subamostradas em levantamentos avifaunísticos, o que constitui um fator que compromete ações para a sua conservação (MOTTA-JÚNIOR, 1996). Assim, como os estudos realizados no estado são poucos, este trabalho teve como objetivo realizar um levantamento das espécies de corujas que compõem a comunidade de Strigiformes em uma área de Mata Atlântica.

\section{Materiais e Métodos}

\section{Área de estudo}

O estudo foi realizado na Reserva Particular do Patrimônio Natural (RPPN) Bugerkopf $\left(27^{\circ} 00^{\prime} \mathrm{S}-\right.$ $49^{\circ} 04^{\prime} \mathrm{W}$ ) (Figura 1), que apresenta uma área de 82,7ha, sendo reconhecida como reserva pela Portaria IBAMA $n^{\circ} 184 \mathrm{~N}$, de 03 de dezembro de 1992, com publicação no Diário Oficial da União de 07 de janeiro de 1993. A RPPN faz limite com o Parque Nacional da Serra do Itajaí.

A cobertura florestal pode ser classificada como Floresta Ombrófila Densa Baixo-Montana, MesoMontana e Alto-Montana, sendo inserida dentro do Domínio Mata Atlântica (KLEIN, 1979). A vegetação sofreu cortes anteriores à criação da Reserva, sendo observadas as seguintes fisionomias sucessionais: floresta primária, com $15 \%$ da área original, sendo encontrada principalmente no terço superior da encosta; floresta primária alterada, cobertura esta perfazendo $75 \%$ da área que sofreu ação extrativista madeireira. A área remanescente apresenta-se em estádios avançados de sucessão ecológica (BRANDT, 2005).

O clima da região é do tipo Mesotérmico Úmido, com temperatura média anual de $21^{\circ} \mathrm{C}$ (GAPLAN, 1986). A precipitação pluviométrica anual varia entre 1600 e $1800 \mathrm{~mm}$, com a média da umidade relativa do ar entre 75 e $80 \%$.

A área está situada a $12 \mathrm{~km}$ do centro da cidade de Blumenau, Santa Catarina. A topografia local é bastante acidentada estando situada entre 95 e 528m acima do nível do mar (BRANDT, 2005). As montanhas destas áreas de conservação pertencem a Serra do Itajaí, incluída na Unidade Geomorfológica da Serra do Tabuleiro. Os agrupamentos rochosos que as constituem são principalmente gnaisses, granitos e migmatitos, que datam de mais de 500 milhões de anos (SANTOS, 1996). 
FIGURA 1: Localização da RPPN Bugerkopf, ao sul do município de Blumenau, SC. Fonte: FURB.

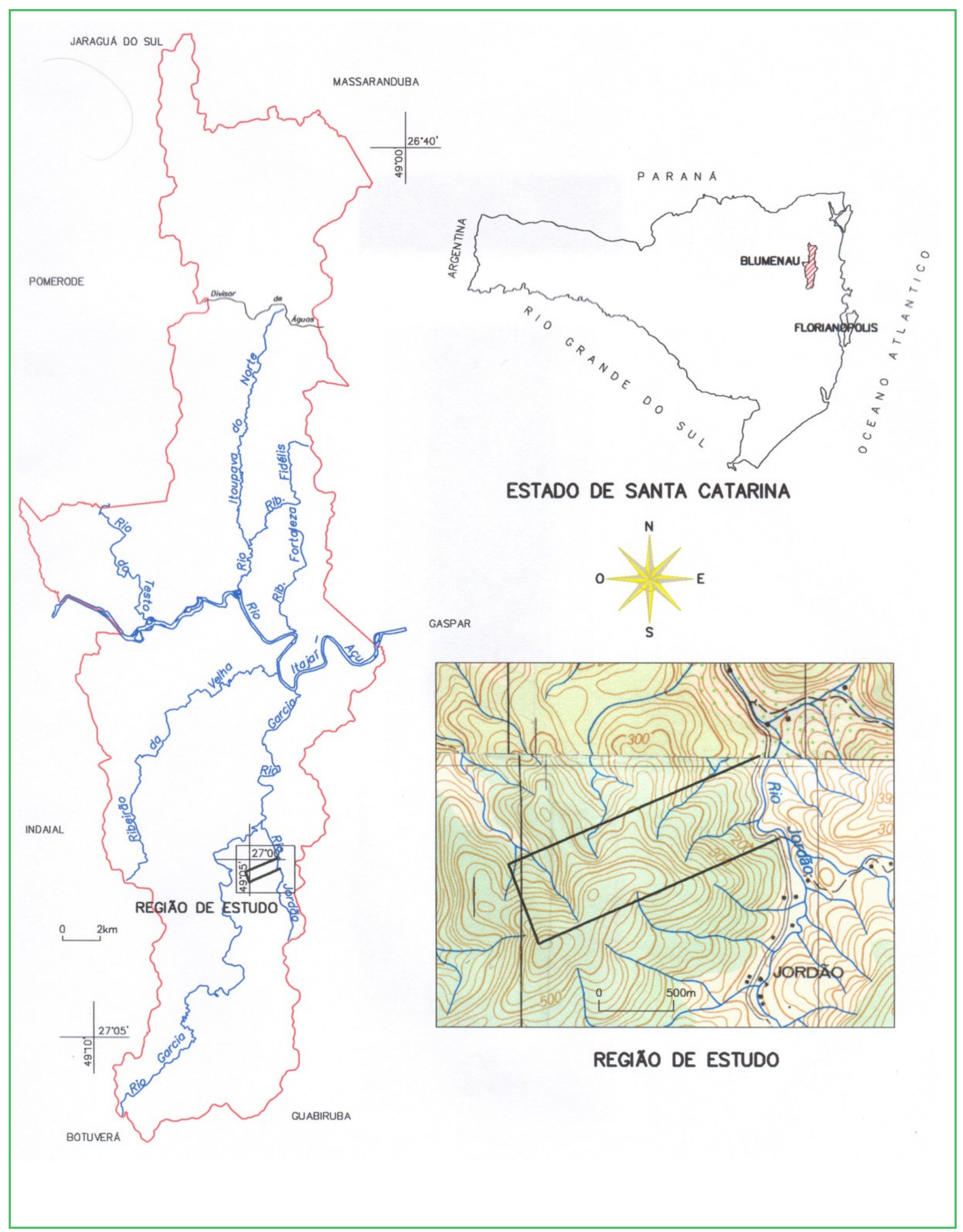




\section{Levantamento de Strigiformes}

Os trabalhos de campo tiveram início em fevereiro de 2005 e foram encerrados em maio de 2006. Para o levantamento das espécies de corujas desta área, foram realizadas mensalmente de duas a três visitas noturnas em fases de lua crescente ou cheia (BRAGA, 2006), entre 18:00 e 23:00 horas, período em que a atividade destas espécies de aves é maior (BORGES et al., 2004). Eventualmente foram feitos registros ao amanhecer, entre 05 e 07:00 horas, utilizando o playback fora dos pontos de amostragem noturna.

Para o registro visual e auditivo das espécies foi percorrida a trilha existente no local e foram selecionados sete pontos de escuta distantes entre si cerca de $200 \mathrm{~m}$ (BORGES et al., 2004; MOTTA-JÚNIOR, 2006), totalizando $1,4 \mathrm{~km}$ na trilha da RPPN. Os pontos de escuta se diferenciaram em relação à cobertura vegetal e de acordo com a proximidade da borda da floresta. Os pontos 1 e 2 localizavam-se próximos a uma nascente e apresentavam boa cobertura vegetal (secundária avançada); o ponto 3 estava localizado em área aberta na Sede da RPPN; os pontos 4 e 5 apresentavam cobertura florestal secundária inicial; os pontos 6 e 7 localizavamse em áreas mais altas e de floresta secundária avançada. Com exceção destes dois últimos pontos, todos os demais estavam próximos à borda do fragmento.

Foi utilizada a técnica do playback das vozes (gritos e cantos) das espécies de corujas possíveis de serem registradas no local, sendo que a sequência de vocalizações das espécies de corujas foi categorizada de acordo com o tamanho das espécies, começando do menor para maior, evitando assim a inibição das pequenas corujas (MOTTA-JÚNIOR, 1996). Foram selecionadas as vozes de 13 espécies corujas relatadas por Rosário (1996) em Santa Catarina: Glaucidium minutissimum, Glaucidium brasilianum, Megascops choliba, Megascops atricapilla, Megascops sanctaecatarinae, Strix virgata, Strix huhula, Strix hylophila, Asio stygius, Asio clamator, Pulsatrix perspicillata, Pulsatrix koeniswaldiana e Bubo virginianus. As gravações das vocalizações são de Hardy et al. (1989), das quais possuem tempo variado de gravação por espécie. A cada reprodução vocal de coruja, que variava de $30 \mathrm{~s}$ a $100 \mathrm{~s}$, seguia-se um intervalo de $60 \mathrm{~s}$. A permanência em cada ponto foi de cerca de $30 \mathrm{~min}$.
Além do playback foram registradas as vocalizações espontâneas, ouvidas durante o deslocamento entre os pontos de escuta. As amostragens foram realizadas em noites sem chuva.

\section{Resultados e Discussão}

O esforço amostral consistiu de $69 \mathrm{~h}$ de campo, percorrendo três pontos de escuta por noite na sequência, desta forma, cada ponto foi amostrado pelo menos uma vez por mês, resultando em 112 pontos com repetição. Durante os trabalhos de campo foram registradas seis espécies de corujas: T. alba (suindara), M. choliba (corujinha-do-mato), M. sanctaecatarinae (corujinhado-sul), P. koeniswaldiana (murucututu-de-barrigaamarela), G. minutissimum (caburé-miudinho) e $A$. stygius (mocho-diabo).

O número de espécies registrado na RPPN Bugerkopf é similar aos resultados obtidos em outros estudos na Mata Atlântica (AMARAL, 2007; LEGAL et al., 2009). Em um levantamento das espécies de aves noturnas na RPPN Chácara Edith, no município de Brusque, Santa Catarina, Legal et al. (2009) registraram cinco espécies de corujas com o método de playback (M. sanctaecatarinae, P. koeniswaldiana, S. virgata, G. minutissimum e A. stygius) em onze pontos fixos, também separados por uma distância de $200 \mathrm{~m}$, em ambientes com área aberta, borda e interior da floresta.

Da mesma forma, em um estudo relacionando a presença de corujas com componentes da estrutura da vegetação em ambiente de Mata Atlântica, Amaral (2007) registrou no Parque Estadual do Rio Doce em Minas Gerais seis espécies: M. choliba, G. minutissium, G. brasilianum, P. koeniswaldiana, S. virgata e $A$. clamator. Os transectos utilizados eram seis de $1500 \mathrm{~m}$ e três de $900 \mathrm{~m}$, com pontos de escuta distantes por $300 \mathrm{~m}$.

Quanto à forma de registro, as corujas detectadas de forma espontânea representaram cerca de 51,8\% dos registros, enquanto o playback representou $48,2 \%$, demonstrando que esta é uma técnica eficiente no estudo de corujas na Floresta Ombrófila Densa. Já na Amazônia Borges et al. (2004) observaram que a maioria das corujas $(77 \%)$ foi detectada de forma espontânea. 
Todos registros de T. alba correspondem a 5 registros espontâneos, principalmente nos pontos mais próximos da sede da RPPN ou na borda da floresta, esta espécie não estava na lista das vocalizações reproduzidas por playback por não se tratar de uma espécie florestal. Como descrevem outros autores (MOTTA-JÚNIOR, 1996; SICK, 1997; DEVELEY; ENDRIGO, 2004), esta espécie está geralmente associada a atividades antrópicas, vivendo e se reproduzindo em cidades e áreas desmatadas.

A corujinha do mato, M. choliba, considerada comum no país (MOTTA-JÚNIOR, 1996; SICK, 1997; BRAGA, 2006) e uma espécie registrada em levantamentos de avifauna geral em Santa Catarina, teve apenas dois registros neste trabalho, um de forma espontânea e outro por playback.

Além de $M$. choliba, outra espécie do gênero foi registrada neste estudo, porém não foi identificada. Foram obtidos três registros mediante uso do playback, após a reprodução de $M$. atricapilla e um espontâneo, totalizando $10 \%$ de frequência de ocorrência. Até recentemente as únicas corujas do gênero conhecidas para Santa Catarina eram $M$. choliba e $M$. sanctaecatarinae. No entanto, a ocorrência de $M$. atricapilla foi recentemente confirmada através da obtenção de gravações de sua vocalização nas partes baixas do litoral de Santa Catarina, como em São Francisco do Sul (XC83862 - número do registro sonoro digital indexado no xeno-canto, www.xenocanto.org), Pomerode (XC88620), e em Praia Grande (BUZZETTI, 2000). Morfologicamente $M$. atricapilla é muito semelhante a $M$. sanctaecatarinae, e mesmo a vocalização do macho desta última é muito semelhante ao canto da primeira, dificultando a identificação.

Considerando que $M$. sanctaecatarinae teve sua vocalização gravada nas partes altas do Parque Nacional da Serra do Itajaí (XC30529; XC41884), e que os registros de $M$. atricapilla em Pomerode são relativamente próximos, optamos por deixar a identidade da coruja registrada e aguardar por uma documentação da vocalização na área de estudo.

Pulsatrix koeniswaldiana é considerada endêmica da MataAtlântica,(DEVELEY; ENDRIGO, 2004; BENCKE et al., 2006). Foi registrado apenas um indivíduo subadulto após o amanhecer acima do ponto sete. Foram um registro espontâneo e um induzido pelo playback.

Glaucidium minutissimum teve o maior número de registros vocais neste estudo, sendo seis espontâneos e sete com playback. Esta espécie respondia rapidamente ao playback, permanecendo em atividade vocal por mais de $15 \mathrm{~min}$. Também foi possível registrá-la após o amanhecer e antes do anoitecer, confirmando seu hábito noturno e diurno (HILTY; BROWN, 1986). Apesar habitar o dossel da floresta, tentou se aproximar ao local em que se reproduzia sua vocalização, entretanto não foi possível sua visualização. O primeiro registro desta espécie para o estado foi apenas na década de 1990 no Morro do Baú (MARTERER, 1996), sendo registrada posteriormente em outras localidades (ALBUQUERQUE; BRÜGGEMANN, 1996; AZEVEDO et al., 2003).

Para A. stygius foram obtidos neste trabalho três registros, dois deles registrados ao amanhecer após a utilização de playback. Esta espécie está na categoria de dados deficientes (DD) de conservação segundo o Livro Vermelho da Fauna Ameaçada no Paraná, devido ao desconhecimento sobre a história natural desta espécie (STRAUBE et al., 2004). Também é considerada uma espécie rara (MOTTA-JÚNIOR, 1996), ou indeterminada devido à escassez de registros (AZEVEDO et al., 2003).

Verificou-se que as espécies de corujas podem apresentar diferentes respostas a playback. Algumas responderam rapidamente e por alguns minutos, como G. minutissimum, ou apenas um único grito após a reprodução de sua vocalização, como M. choliba.

A continuidade e o aprimoramento de estudos que possam ajudar a identificação precisa de Strigiformes, através de suas vocalizações, permitirão uma melhor compreensão sobre as espécies da Ordem Strigiformes. Neste estudo, o gênero Megascops se mostrou de difícil identificação a nível específico através de suas vocalizações, principalmente porque o ruído de uma nascente impossibilitou a gravação das vocalizações.

Os poucos registros de corujas neste estudo podem ser relacionados às vocalizações utilizadas no playback, já o ideal seria a gravação e a reprodução de vozes de espécimes encontrados na região. 


\section{Agradecimentos}

Somos gratos aos proprietários da RPPN Bugerkopf, Sra. Edela Bacca e Sr. Lauro Eduardo Bacca por permitirem a pesquisa em sua propriedade. Aos amigos que foram a campo: Cristiane Krieck, Michele Regina de Jesus Novaes, Gregori Thom e Josias Alan Resini. Aos revisores deste manuscrito pelas valorosas sugestões e correções.

\section{Referências}

ACCORDI, I. A.; BARCELOS, A. Novas ocorrências e registros notáveis sobre distribuição de aves em Santa Catarina, sul do Brasil. Biotemas, Florianópolis, v. 21, n. 1, p. 85-93, 2008.

ALBUQUERQUE, J. L. B.; BRÜGGEMANN, F. M. A avifauna do Parque Estadual da Serra do Tabuleiro, Santa Catarina, Brasil e as implicações para a sua conservação. Acta Biológica Leopoldensia, São Leopoldo, v. 18, n. 1, p. 47-68, 1996.

AMARAL, K. F. Composição e abundância de corujas em Floresta Atlântica e sua relação com variáveis de habitat. 2007, 47 f. Dissertação (Mestrado em Ecologia) - Universidade Federal do Rio Grande do Sul, Porto Alegre, 2007.

AZEVEDO, M. A. G.; MACHADO, D. A.; ALBUQUERQUE, J. L. B. Aves de rapina na Ilha de Santa Catarina, SC: composição, freqüência de ocorrência, uso de habitat e conservação. Ararajuba, Londrina, v. 11, n. 1, p. 75-81, 2003.

BENCKE, G. A.; BENCKE, C. S. C. The potencial importance of road deaths as a cause of mortality for large forest owls in southern Brazil. Cotinga, Cape May Point, v. 11, p. 17-18, 1999.

BENCKE, G. A.; MAURICIO, G. N.; DEVELEY, P. F.; GOERCK, J. M. Áreas importantes para a conservação das aves do Brasil. Parte I - Estados do Domínio da Mata Atlântica. São Paulo: SAVE Brasil, 2006. $494 \mathrm{p}$.

BORGES, S. H.; HENRIQUES, L. M.; CARVALHAES, A. 2004. Density and habitat use by owls in two Amazonian forest types. Journal Field Ornithology, Lawrence, v. 75, n. 2, p. 176-182, 2004.

BRANDT, C. S. Comunidade de aves de sub-bosque em um trecho de floresta ombrófila densa de encosta, na região de Blumenau/Santa Catarina. 2005. 57 f. Monografia (Graduação Ciências Biológicas) - Universidade Regional de Blumenau, Blumenau, 2005.

BRAGA, A. C. Uso de habitat da corujinha-do-mato Megascops choliba e da coruja-buraqueira Athene cunicularia (Strigiformes: Strigidae) em remanescentes de cerrado da região central do Estado de São Paulo. 2006. 92 f. Dissertação (Mestrado em Ecologia) - Universidade de São Paulo, São Paulo. 2006.

BUZZETTI, D. R. C. Distribuição altitudinal de aves em Angra dos Reis e Parati, sul do estado do Rio de Janeiro, Brasil. In: ALVES, M. A. S.; DA SILVA, J. M. C.; SLUYS, M. V.; BERGALLO, H. G.; ROCHA, C. F. D. (Ed.). A Ornitologia no Brasil - Pesquisa Atual e Perspectivas. Rio de Janeiro: EDUERJ, 2000. p. 131-148.
CRBO - COMITÊ BRASILEIRO DE REGISTROS ORNITOLÓGICOS. 2011. Listas das aves do Brasil. 10 ed. Disponível em <http://www.cbro.org.br>. Acesso em: 12 out. 2011. DEVELEY, P. F.; ENDRIGO, E. Aves da Grande São Paulo: guia de campo. São Paulo: Ed. Aves e fotos, 2004. 295 p.

GAPLAN. Gabinete de Planejamento e Coordenação Geral. Rio de Janeiro: Aerofoto Cruzeiro. 1986. 173 p.

HARDY, J. W.; COFFEY JR, B. B.; REYNARD, G. B. Voices of the New World Owls. Gainesville: ARA Records (ARA 16), 1989. s/ paginação.

HILTY, S. L.; BROWN, W. L. A guide to the birds of Colombia. Princeton: Princeton University, 1986. 836 p.

KLEIN, R. M. Ecologia da flora e vegetação do Vale do Itajaí. Sellowia, Itajaí, v. 31, p. 1-164, 1979.

KÖNIG, C.; WEICK, F. Owls of the world. A guide to the owls of the world. 2 ed. London: Christopher Helm, 2008. 512 p.

LEGAL, E.; CADORIN, T. J.; KOHLER, G. Strigiformes e Caprimulgiformes em Santa Catarina, sul do Brasil: registros relevantes e novas localidades. Biotemas, Florianópolis, v. 22, n. 4, p. 125-132, 2009.

MARTERER, B. T. P. Avifauna do Parque Botânico do Morro do Baú. Florianópolis: FATMA, 1996. 74 p.

MOTTA-JÚNIOR, J. C. Ecologia alimentar de corujas (Aves: Strigiformes) na região central do estado de São Paulo: biomassa, sazonalidade e seletividade de suas presas. 1996. 119 f. Tese (Doutorado em Ecologia) - Universidade Federal de São Carlos, São Carlos, 1996.

MOTTA-JÚNIOR, J. C. Relações tróficas entre cinco Strigiformes simpátricas na região central do Estado de São Paulo. Revista Brasileira Ornitologia, São Leopoldo, v. 14, n. 4, p. 359-377, 2006.

PIACENTINI, V. Q.; GHIZONI-JR, I. R.; AZEVEDO, M. A. G.; KIRWAN, G. M. Sobre a distribuição de aves em Santa Catarina, Brasil, parte 1: registros relevantes para o estado ou inéditos para a Ilha de Santa Catarina. Cotinga, Cape May Point, v. 26, p. 25-31, 2006.

ROSÁRIO, L. A. As aves de Santa Catarina. Florianópolis: Fatma, 1996. 326 p.

RUPP,A.E.;BRANDT, C. S.;FINK, D.; THOME SILVA, G.; LAPS, R. R.; ZIMMERMANN, C. E. Registros de Caprimulgiformes e a primeira ocorrência de Caprimulgus sericocaudatus (bacurau-rabode-seda) no Estado de Santa Catarina, Brasil. Revista Brasileira de Ornitologia, São Leopoldo, v. 15, n. 4, p. 605-608, 2007.

SANTOS, G. F. Vale do Garcia (Blumenau - SC): análise climatogeomorfológica e a repercussão dos episódios pluviais no espaço urbano. 1996. 362 f. Tese (Doutorado em Geografia) Universidade de São Paulo, São Paulo. 1996.

SICK, H. Ornitologia brasileira: uma introdução. Rio de Janeiro: Nova Fronteira. 1997. 823 p.

SILVA, J. M. C.; COELHO, G.; GONZAGA, L. P. Discovered on the brink of extintion: a new species of Pygmy-Owl (Strigidae: Glaucidium) from Atlantic Forest or northeastern Brazil. Ararajuba, Londrina, v. 10, n. 2, p. 123-130. 2002.

STRAUBE, F. C.; URBEN-FILHO, A.; KAJIWARA, D. Aves. In: MIKICH, S. B.; BÉRNILS, R. S. (Ed.). Livro vermelho da fauna ameaçada no estado do Paraná. Curitiba: Instituto Ambiental do Paraná, 2004. p. 143-496. 